\title{
DISCRIMINATION OF URINARY STRAINS OF ESCHERICHIA COLI BY FIVE TYPING METHODS
}

\author{
D. C. Old, Pamela B. Crichton, Angela J. Maunder \\ and Margaret I. Wilson \\ Department of Bacteriology, University of Dundee Medical School, Ninewells Hospital, Dundee \\ DD1 9SY, Scotland
}

SEROTYPING is probably the most widely used method for typing strains of Escherichia coli, but the range of antisera required restricts its use to a few specialised laboratories. Other typing methods are available, and the purposes of this investigation were to evaluate the techniques of biotyping (Crichton and Old, 1979), resistotyping (Elek and Higney, 1970) and haemagglutinin typing (Duguid, Anderson and Campbell, 1966; Duguid, Clegg and Wilson, 1979), to assess their convenience for use in routine diagnostic laboratories, and to determine whether combinations of these methods would lead to more precise identification of sequential isolates of $E$. coli from patients with urinary-tract infections.

\section{MATERIALS AND METHODS}

Bacteria. The 156 cultures of E. coli investigated were isolated from 1976 to 1979 in the Bacteriology Department, Ninewells Hospital, Dundee, from mid-stream or catheter specimens of urine from four male patients (nos. 2, 4, 8, and 20) aged from 5 months to 76 years, and from 16 female patients, aged from 23 to 86 years; all had "significant bacteriuria", i.e., at least $10^{5}$ bacteria/ml of freshly voided urine. From some, only two cultures were isolated from specimens taken a few days apart, whereas from others multiple specimens yielded cultures over periods of up to 2 years.

The 15 reference strains used for standardisation of the resistotyping chemicals were obtained from the Bacteriology Department, St George's Hospital Medical School, London and included 13 of the 16 reference strains used in the original resistotyping scheme (Elek and Higney, 1970). Strains were stored on Dorset's egg slopes at ambient temperature.

Culture media. The liquid medium used was Nutrient Broth No. 2 (Oxoid Ltd, Basingstoke, Hants) and phosphate-buffered agar (PBA) was the above broth with $\mathrm{KH}_{2} \mathrm{PO}_{4} 3.5 \mathrm{~g}, \mathrm{Na}_{2} \mathrm{HPO}_{4}$ $6.5 \mathrm{~g}$, and Bacto Agar (Difco Laboratories, West Molesey, Surrey) $15 \mathrm{~g} /$ litre $(p \mathrm{H} \mathrm{7.0)}$. MacConkey Agar was from Oxoid. For resistotyping we used Antibiotic Agar Medium No. 2 (Oxoid), prepared in $0.1 \mathrm{M}$ tris(hydroxymethyl)-methylamine- $\mathrm{HCl}(p \mathrm{H} \mathrm{7.6)}$. Sensitivity agar was DST Agar (Oxoid) with $5 \%$ (v/v) lysed horse-blood.

Biotyping. Biotyping media were prepared and tests performed and interpreted according to the criteria of Crichton and Old (1979). There was a close correlation between sucrose and raffinose fermentation, and because we found the latter test to be the more reliable, results for sucrose fermentation have been omitted from the tables but are presented in the text when they are significant for strain identification. 
Resistotyping. The concentrations ( $\mathrm{w} / \mathrm{v}$ ) of the stock solutions of sodium arsenate (chemical A) $0.75 \%$, phenylmercuric nitrate (B) $0.0006 \%, 4: 4^{\prime}$-diamidinodiphenylamine dihydrochloride (C) $0.005 \%$, boric acid (D) $3 \%$ and copper sulphate (G) $0.7 \%$ were those of Elek and Higney (1970); the concentrations of acriflavine (E) $0.009 \%$, 4-chloresorcinol (F) $0.65 \%$ and malachite green $(\mathrm{H}) 0.0011 \%$ were those that, in our hands, allowed correct assignment of the reference strains as sensitive or resistant to chemicals $\mathrm{E}, \mathrm{F}$ and $\mathrm{H}$. Except for chemicals $\mathrm{B}$ and $\mathrm{F}$ that were dissolved in, respectively, $40 \%$ and $10 \%$ (v/v) ethyl alcohol, the preparation of the stock chemicals followed Elek and Higney (1970). Fresh working solutions were prepared every 5 days because changes in the toxicity of some chemicals occurred during storage.

To ensure that we achieved optimal differentiation of strains by each antibacterial agent, 20 test cultures and five reference strains were inoculated on every plate. The reference strains generally used were 4, 5, 7 and 8 (Elek and Higney, 1970); our strain PC42849 was more satisfactory than any of the above-mentioned 15 reference strains for detection of acriflavine sensitivity. Apart from these modifications, we followed the technique, and described the growth patterns obtained, as outlined by Elek and Higney (1970); thus, ABDE(F) indicated that the strain was resistant to chemicals A, B, D and E, partially resistant to chemical F and sensitive to chemicals $\mathrm{C}, \mathrm{G}$ and $\mathrm{H}$. In addition, by Elek and Higney's criteria, cultures differing in their description by one bracket, e.g., $\mathrm{AD}(\mathrm{E}) \mathrm{GH}$ and $\mathrm{ADEGH}$, were regarded as identical.

Production and detection of haemagglutinins. For the production of type-1 fimbriae and mannose-sensitive (MS) haemagglutinins, bacteria were grown serially at $37^{\circ} \mathrm{C}$ in static broths $(10 \mathrm{ml})$ in test tubes stoppered with cotton wool (Duguid et al., 1955), and for mannose-resistant and eluting (MRE) haemagglutinins, at $37^{\circ} \mathrm{C}$ for $24 \mathrm{~h}$ on PBA (Duguid et al., 1979). Bacterial suspensions were tested with erythrocytes prepared by the methods of Duguid et al. (1979). For the detection of MRE haemagglutinins, however, only seven species of erythrocytes were used: fowl (F), guinea-pig (G), horse (H), man group $O(M)$, ox $(\mathrm{O})$, pig $(\mathrm{P})$ and sheep $(\mathrm{S})$. Cultures producing MRE haemagglutinins that reacted, for example, with the erythrocytes of man, pig and sheep, were designated type MPS.

Serological analysis. The $\mathrm{O}$ serogroup was determined as described by Crichton and Old (1979). The 24 commercial antisera (Wellcome Reagents Ltd, Beckenham, Kent) were urinary types $\mathrm{O} 1, \mathrm{O} 2, \mathrm{O} 4, \mathrm{O} 5, \mathrm{O} 6, \mathrm{O} 9, \mathrm{O} 11, \mathrm{O} 18, \mathrm{O} 39$ and $\mathrm{O} 75$, and enteropathogenic types $\mathrm{O} 26, \mathrm{O} 44$, O55, O86, O111, O112, O114, O119, O124, O125, O126, O127, O128 and 0142. Fourteen cultures from patient no. 10 were serotyped by Dr B. Rowe, Division of Enteric Pathogens, Colindale Avenue, London.

Antibiotic-sensitivity tests were performed on lysed horse-blood agar by the methods of Stokes (1975) and with the antibiotics available on Oxoid Multodisk No. 2863E: ampicillin (25 $\mu \mathrm{g})$, cephaloridine $(25 \mu \mathrm{g})$, triple suphonamide $(500 \mu \mathrm{g})$, kanamycin $(30 \mu \mathrm{g})$, nalidixic acid (30 $\mu \mathrm{g})$, nitrofurantoin $(200 \mu \mathrm{g})$, co-trimoxazole $(25 \mu \mathrm{g})$ and tetracycline $(50 \mu \mathrm{g})$. Cultures were judged to be resistant to an antibiotic when growth occurred within $8 \mathrm{~mm}$ around the edge of the disc after overnight incubation.

\section{RESULTS}

\section{Patients yielding repeated isolates of the same strain}

All characters identical. Each culture from each of the six patients, nos. 1, $2,3,4,5$ and 6 , showed the biotype, resistotype and haemagglutinin type characteristic of that patient's organism and in each series the strains were of the same serotype, or non-typable, and showed the same antibiogram patterns (table I).

Variation in some characters. In another four patients, minor variations in some typing characters were observed (table I). Thus, all six cultures from patient no. 7 were resistant to ampicillin, sulphonamides and co-trimoxazole but the final four were also nalidixic acid resistant. However, they were 


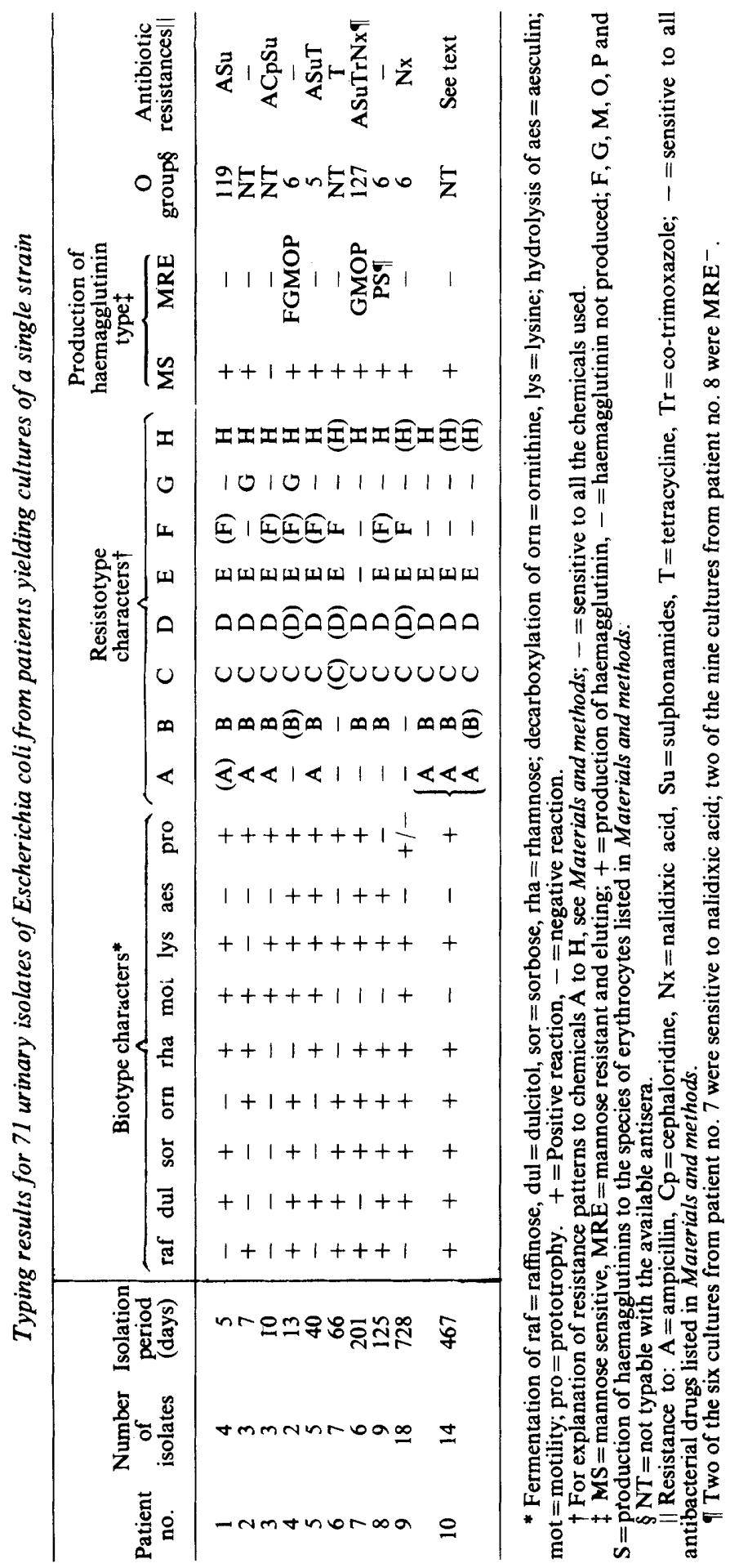


identical in biotype, resistotype and haemagglutinin type $\left(\mathrm{MS}^{+} \mathrm{MRE}^{+}\right.$; type GMOP).

Nine strains of $E$. coli isolated during a period of 125 days from patient no. 8 , who had a two-year history of urinary-tract infection, were all nicotinamide auxotrophs of the same biotype, resistotype and serogroup (06), and were $\mathrm{MS}^{+}$. However, the first and fifth cultures were $\mathrm{MRE}^{-}$variants that did not form the MRE (type PS) haemagglutinins produced by the other seven cultures. All 18 strains from patient no. 9, who had a staghorn calculus in her only kidney, were of the same resistotype, haemagglutinin type $\left(\mathrm{MS}^{+} \mathrm{MRE}^{-}\right)$ and serogroup (O6), and were resistant to nalidixic acid and although their biotype profiles were similar, the eight cultures isolated in the first 70 days were prototrophs, whereas the subsequent 10 were thiamine auxotrophs.

There were minor variations among the 14 strains isolated during 467 days from patient no. 10. Their biotypes were identical except for two isolates (days 435 and 446) that gave motile variants. All the strains were $\mathrm{H}^{-}$and were untypable with available $\mathrm{O}$ and $\mathrm{K}$ antisera at the Division of Enteric Pathogens, Colindale (Dr B. Rowe, personal communication). Although cultures of resistotype $\mathrm{ABCDE}(\mathrm{H})$ (days 20, 217 and 435) showed acceptable strain identity with those of resistotypes $\mathrm{ABCDEH}$ (days 1, 86, 98, 216, 218, 219, 267, 281, 446 and 447) and $\mathrm{A}(\mathrm{B}) \mathrm{CDE}(\mathrm{H})$ (day 313), differences of two partial resistances among cultures of the latter resistotypes would ordinarily suggest non-identity of strains. All cultures were resistant to ampicillin, cephaloridine, nalidixic acid, sulphonamides and co-trimoxazole; seven of them (days $216,217,218,219,435,446$ and 447) were also resistant to nitrofurantoin. All were $\mathrm{MS}^{+} \mathrm{MRE}^{-}$. The slight variations among these isolates seem insufficient to indicate that distinct strains were involved, especially because all of the isolates had the same, rare, Suc ${ }^{-} \mathrm{Raf}^{+}$phenotype.

\section{Patients each yielding isolates of different strains}

Table II, which presents our typing results for the 85 urinary isolates of $E$. coli from another 10 patients (nos. 11-20), indicates that the sequential isolates from their urines represented different strains, that showed marked differences in more than one of the typing characters.

Although the data in table II are largely self explanatory, we draw attention to the following points: (i) from patient no. 14, only one of the four cultures of the strain isolated during days 109-244 was $\mathrm{MRE}^{+}$(type GMOP); (ii) the biochemically identical first, fifth and seventh cultures from patient 17 (days 1 , 114 and 262) were of resistotypes so different, viz., $\mathrm{ACDE}(\mathrm{H}),(\mathrm{A})(\mathrm{B})(\mathrm{D}) \mathrm{EFH}$ and $A C D(E) F(H)$, that they probably represented distinct strains; (iii) from patient no. 18, a culture of distinct colonial appearance was isolated on day 140 , and although it was of similar biotype and haemagglutinin type (MS $^{+} \mathrm{MRE}^{+}$, type MPS) to the other isolates it showed a major resistotype difference in character C; (iv) from patient no. 19, five cultures of the same serogroup $(\mathrm{O} 4)$ and resistotype were isolated but, whereas the first two cultures did not ferment sorbose and were non-haemagglutinating, the other three 


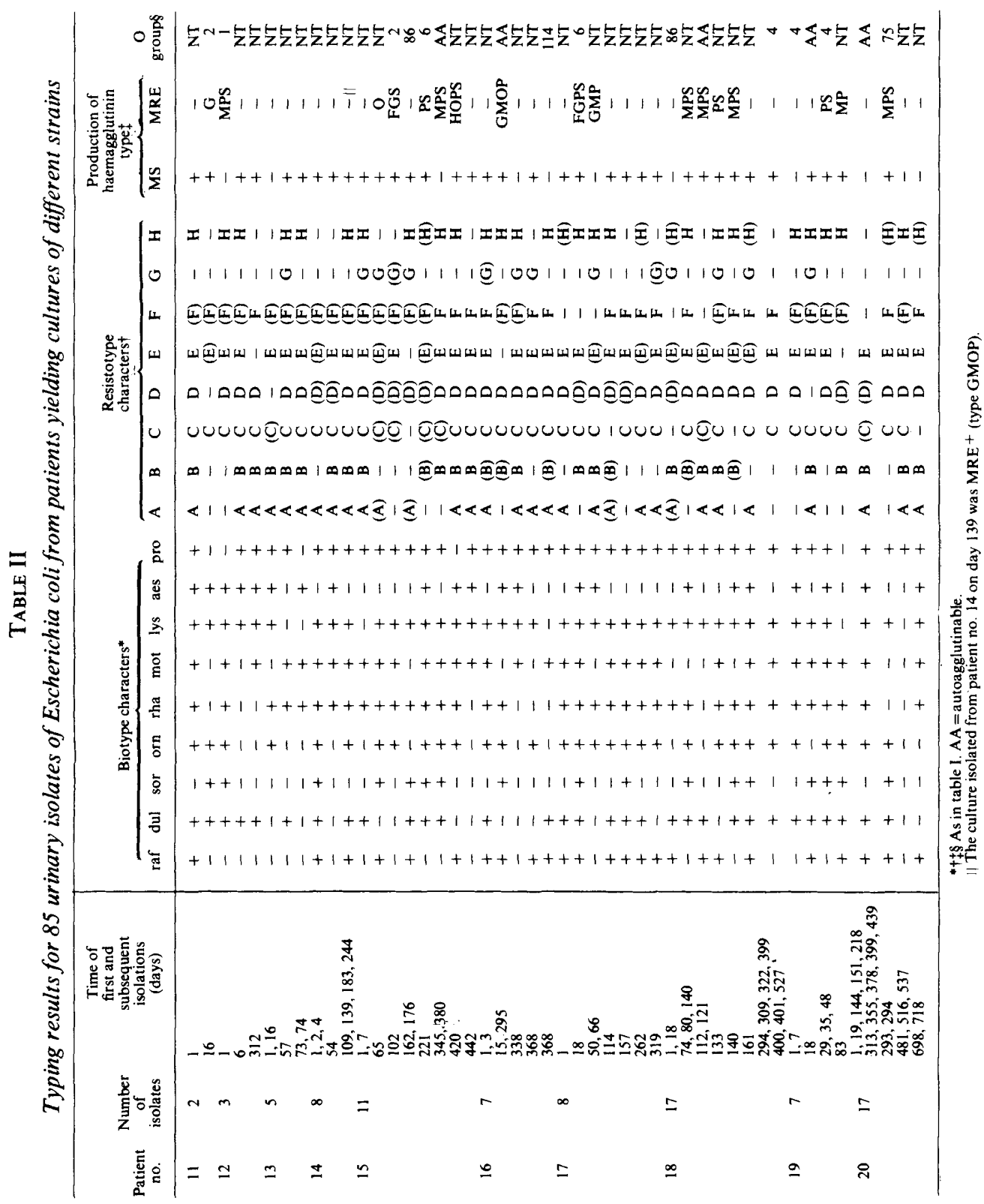


fermented sorbose and were haemagglutinating (MS+ $\mathrm{MRE}^{+}$, type PS); (v) from patient no. 20 , at least four distinct strains were isolated during nearly 2 years; when the patient was septicaemic (day 698), a strain was isolated from his blood, indistinguishable from the strain then present in his urine (days 698 and 718).

\section{Discussion}

Although typing by complete serological analysis of the somatic (O), capsular $(\mathrm{K})$ and flagellar $(\mathrm{H})$ antigens of $E$. coli provides accurate strain identification, it is not practicable for most laboratories. With a limited range of 24 commercial $\mathrm{O}$ sera, we typed only $68(43.6 \%)$ of the 156 cultures. The most common types were O6 (31 cultures from five patients) and O4 (12 cultures from two patients). The remaining 88 cultures were untypable $(45.5 \%)$ or autoagglutinable $(10.9 \%)$. Thus, limited $\mathrm{O}$ serotyping was of little help in characterising most of the strains.

On the other hand, we were able to assign to each culture a resistotype profile and a biotype profile; generally, the agreement between the results from the two systems was good. The resistotyping scheme was highly discriminatory and we recognised 47 different resistotype profiles among 156 isolates from 20 patients. The stability of the resistotype characters was shown by our findings that repeated testing of the cultures never revealed more than a minor character variation even when the replicate tests were made at considerable intervals of time by different members of our group. Similarly, we recognised 43 different biotype profiles among the 156 cultures and showed that the results from replicate testing of cultures were highly reproducible. The discrimination and reproducibility of biotyping and resistotyping suggest that they would be valuable in laboratories in which serotyping is not convenient, and the introduction of either method in addition to $O$ serotyping should considerably improve strain identification; for example, among the 31 cultures of $O$ group 6 examined, five biotypes and five resistotypes were noted and the biotype/resistotype combination of the O6 cultures from each of the five patients was different.

The majority $(80 \%)$ of the 156 cultures produced MS haemagglutinins with a constant red-cell specificity. The production of type- 1 fimbriae, determining MS activity, is a stable, chromosomal property that may provide an additional discriminatory biotype marker. It has been found in $76 \%$ of a larger series of 387 strains of E. coli of diverse origin (Duguid et al., 1979).

Among the $42 \mathrm{MRE}^{+}$cultures of $E$. coli, we noted 11 distinct MRE patterns. Although the MRE specificity often supported the other typing data, it was not itself stable enough for epidemiological purposes. Thus, among some series of cultures, otherwise identical in biotype, resistotype, MS type, serotype and antibiogram type, differences in MRE status were sometimes noted (e.g., patient no. 8). Most MRE cultures of $E$. coli are fimbriate (Duguid et al., 1979) with properties similar to haemagglutinating E. coli cultures with surface antigens K88, K99, CFAI or CFAII, known to be plasmid determined (see Duguid and Old, 1980). Thus, if MRE production is 
similarly controlled, plasmid loss or gain will influence the MRE status of a culture.

It was not the purpose of this paper to discuss the clinical significance of the recovery of the same or different strains from sequential urines from patients with urinary-tract infection, but to investigate means of identifying them by techniques other than serotyping. Our results indicate that series of cultures of $E$. coli from patients can be discriminated by biotyping or resistotyping with considerable accuracy. Because we found biotyping to be technically simpler than resistotyping, we have favoured biotyping as a primary typing method and resistotyping as a secondary method.

Most characters, however, including those of biotyping and resistotyping, are subject to variation during spread of a clone so that sometimes, especially when a single typing method has been used, it is difficult to know the exact relationships between strains. This is particularly so when variation in a typing character comes about by loss or gain of a type-determining plasmid or bacteriophage. For that reason, we feel that it is important to use as many techniques as possible for the characterisation of strains. We will report elsewhere our typing studies on $E$. coli from diverse clinical sources.

\section{SUMMARY}

A series of 156 cultures of Escherichia coli isolated from sequential urines from 20 patients with urinary-tract infection was examined by biotyping, resistotyping, haemagglutinin typing, $O$ serotyping and antibiogram typing. From 10 of the patients, we repeatedly isolated cultures of a single strain; from each of the other 10 patients, different strains were discriminated.

All, cultures were typable by biotyping and resistotyping, and those techniques should prove practicable for laboratories not able to perform complete serological analysis. Only a minority $(43 \cdot 6 \%)$ of the cultures was typed with a limited range of 24 commercial $O$ sera.

Maximum discrimination of strains was achieved by the combined use of several of these techniques.

We thank Professor J. P. Duguid for helpful discussions and Dr B. Rowe for serotyping some of the strains.

\section{REFERENCES}

Crichton, P. B. ANd OLd, D. C. 1979. Biotyping of Escherichia coli. J. med. Microbiol., 12, 473.

Duguid, J. P., ANDerson, E. S. AND Camprell, I. 1966. Fimbriae and adhesive properties in salmonellae. J. Path. Bact., 92, 107.

Duguid, J. P., ClegG, S. AND Wilson, M. I. 1979. The fimbrial and non-fimbrial haemagglutinins of Escherichia coli. J. med. Microbiol., 12, 213.

Duguid, J. P. AND OLD, D. C. 1980. Adhesive properties of Enterobacteriaceae. In Bacterial adherence (receptors and recognition, series B, vol. 6), edited by E. H. Beachey. Chapman and Hall: London, chapter 7. 
Duguid, J. P., Smith, I. W., Dempster, G. And Edmunds, P. N. 1955. Non-flagellar filamentous appendages ("fimbriae") and haemagglutinating activity in Bacterium coli. J. Path. Bact., 70, 335.

ELEK, S. D. AND Higney, L. 1970. Resistogram typing-a new epidemiological tool: application to Escherichia coli. J. med. Microbiol., 3, 103.

STOKES, E. J. 1975. Clinical bacteriology, 4th ed. Arnold: London, p. 217. 